\title{
Functional Nanoparticle-Based Proteomic Strategies for Rapid Characterization of Pathogenic Bacteria
}

\author{
Wei-Jen Chen, ${ }^{1}$ Pei-Jane Tsai, ${ }^{2}$ Yu-Chie Chen ${ }^{* 1}$ \\ ${ }^{1}$ Department of Applied Chemistry, National Chiao Tung University, \\ Hsinchu 300, Taiwan \\ ${ }^{2}$ National Laboratory Animal Center, National Applied Research Laboratories, \\ Taipei 115, Taiwan
}

\section{Supporting Information}

Figure S1 presents the MS/MS spectrum by selecting the peak at m/z 1603, which is derived from Shigella sonnei. The inset displays the peptide sequence that was obtained by combing the MS/MS result with protein database searching. The searching result confirmed that the peak at $\mathrm{m} / \mathrm{z} 1603$ is derived from elongation factor Tu of S. sonnei. Fragment peaks ( $/ \mathrm{N}>3$ ) resulting from precursor ions $(\mathrm{S} / \mathrm{N}>3)$ were submitted via Biotools (v. 3.0) to MASCOT (www.matrixscience.com) using the following search parameters: the database searched was NCBInr 20080616; taxonomy was limited to eubacteria; the enzyme was trypsin; MS and MS/MS tolerances were set at \pm 0.5 and $\pm 0.8 \mathrm{Da}$, respectively; the number of missed cleavages was set to 1 ; the significance threshold was set at $\mathrm{p}<0.05$ because the score was over 50 .

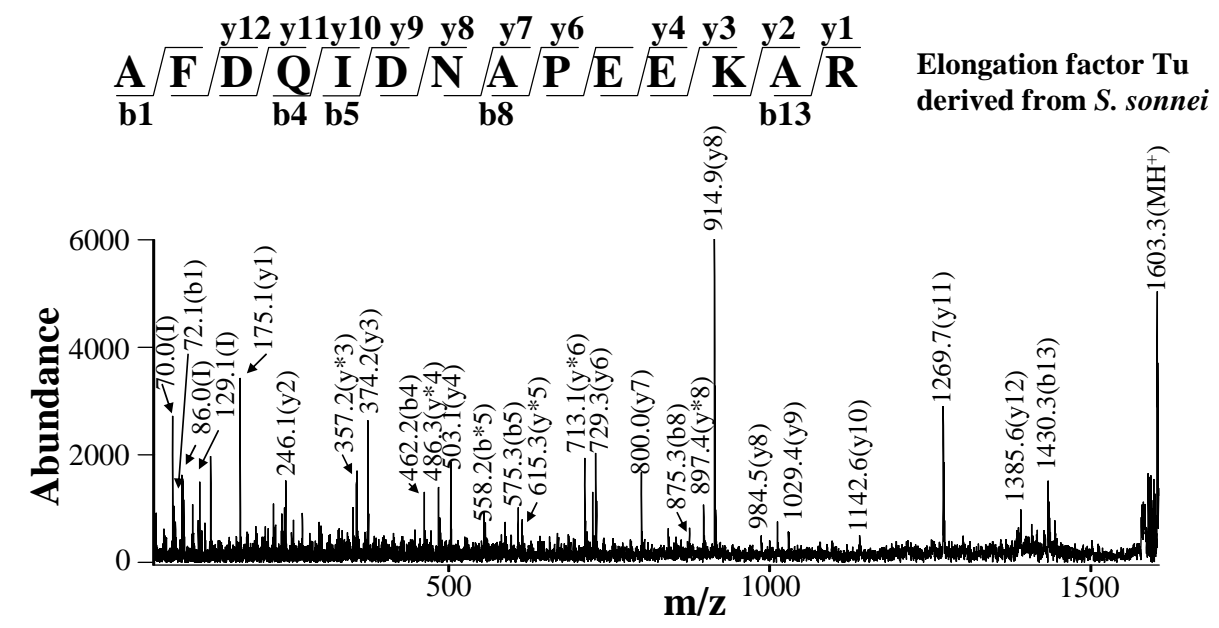

Figure S1. MS/MS spectrum of the ion at m/z 1603 and its corresponding peptide sequence obtained via protein database searching. 